\title{
R. A. R. Gurung: Health Psychology: A Cultural Approach
}

\author{
Wadsworth, Cengage Learning, Belmont, California, 2010, 2nd edn, 518 pp, \\ $\$ 134.95$ (hardbound)
}

\author{
Bruce A. Barron
}

Published online: 6 December 2009

(C) Springer Science+Business Media, LLC 2009

\begin{abstract}
Although employee wellness programs have materialized as an important workplace strategy to optimize employee health and productivity during the last decade, the success of these programs have been dependent on a number of variables [1]. Many programs have placed increasing emphasis on employee emotional well being. As a result health psychology has become an essential component of most corporate wellness programs because it speaks to the potential impact of thought, behavior, and emotions on employee health, illness, and productivity [2]. Cultural diversity is another factor influencing the outcomes of worksite wellness programs [3]. Given the potential impact of health psychology and cultural diversity on the outcomes of employee health and wellness programs in industry today, the publication of Health Psychology: A Cultural Approach, 2nd Edition could not have been more timely.
\end{abstract}

The author's goals for this second edition were to study health and illness from a psychological and cultural perspective and to introduce concepts and practices pertinent to the field of health psychology [4]. Health Psychology is comprised of 14 logically sequenced chapters, most of which are applicable to the work environment, e.g. health psychology, cultural approaches to health, stress across cultures, models of behavior change, health behaviors, pain, and others. The content of each chapter is evidencebased and includes numerous references to the scientific literature. The second edition contains citations to more than 1,000 new research articles in addition to an abundant number of valid annotated web resources. The author also presents real-world case scenarios that enable the reader to

B. A. Barron ( $\square)$

Division of Occupational and Environmental Medicine, University of Rochester Medical Center, Rochester, NY, USA

e-mail: bruce_barron@urmc.rochester.edu learn and understand basic theories, concepts, and principles. Each chapter ends with a succinct summary that reviews essential terms and concepts. The chapter summary includes ten multiple choice questions to test the reader's knowledge and understanding of the material.

In general the text is comprehensive, evidence-based, informative, and written in a style that is both readable and easy to understand. Health Psychology is a robust book that reviews the biopsychosocial approach to health and wellness from a cultural perspective. The author emphasized the importance and potential impact of an individual's culture, i.e. ethnicity, race, age, gender, religion, and family, on health and illness behavior. It is likely that application of the more comprehensive biopsychocultural model rather than more circumscribed biopsyhcosocial model of health will yield better outcomes and greater returns on investment as it relates to employee health and wellness programs. Although probably designed and published for an undergraduate population, the information presented in Health Psychology could easily be applied to the development of workplace wellness programs; therefore, occupational health professionals will want to read Health Psychology and have it available for future reference.

\section{References}

1. Goetzel RZ, Ozminkowski RJ. Health and productivity management: emerging opportunities for health promotion professionals for the 21st century. Am J Health Promot. 2000;14:211-4.

2. Rodin J, Salovey P. Health psychology. Am Rev Psychol. 1989;40: 533-79.

3. Thompson SE, Smith BA, Bybee RF. Factors influencing participation in worksite wellness programs among minority and underserved populations. Fam Community Health. 2005;28(3):267-73.

4. Gurung, RAR. Health psychology: a cultural approach. 2nd ed. Belmont, California: Wadsworth, Cengage Learning; 2010: xviii. 